Memories of the College of Science, University of Kyoto, Series A,

Vol. XXVI, Mathematics No. 2, 1950.

\title{
Note on the Intersection of an Algebraic Variety with the Generic Hyperplane.
}

By

\author{
Yosikazu NAKAI
}

(Receivcel August 20, 1950.)

Let $V^{r}(r>1)$ be an algebraic variety immersed in the projective space $L^{N}, k$ a field of definition for $V$, and $H^{N-1}$ a generic hyperplane with respect to $k$ in $L$. Then it is well known that the intersection $H \cdot V$ is defined and is an irreducible variety ${ }^{1)} W^{r-1}$. In this case the question arises that the variety may get a new singular point other than that of $V$. When the characteristic $p$ of the universal domain is 0 , we know that such a case cannot take place by the second theorem of Bertini. In this note we shall show that it is also true even when the case $p \neq 0$.

To discuss this problem it is sufficient to consider the case when $V$ is lying in the affine space $S^{N}$.

Let

$$
F(X)=\sum_{i=1}^{N} u_{i} X_{i}-v=0 .
$$

be the defining equation for $H$, where $\left(u_{i}, v\right)$ are $(N+1)$-independent variables over $k$ and $X_{i}$ denote the indeterminates of $S$. Let $P=\left(x_{1}, \ldots \ldots, x_{N}\right)$ be any point on $W$, which is simple on $V$. Then we can choose $(N-r)$ polynomials $G_{\nu}(X)(\nu=1, \ldots \ldots, N-r)$ from the definig ideal of $V$ over $k$ such that the rank of the matrix

$$
A=\left(\frac{\partial G_{\nu}}{\partial x_{i}}\right) \quad\left(\begin{array}{l}
\nu=1, \ldots \ldots, N-r \\
i=1, \ldots \ldots, N
\end{array}\right)
$$

is equal to $N-r$. Then the simplicity of $P$ on $W$ can be concluded if we can show that the rank of the matrix 


$$
B=\left(\begin{array}{c}
\frac{\partial G_{\nu}}{\partial x_{i}} \\
u_{i}
\end{array}\right) . \quad\left(\begin{array}{l}
\nu=1, \ldots \ldots, N-r \\
i=1, \ldots \ldots, N
\end{array}\right)
$$

is equal to $N-r+1$.

Let $\operatorname{dim}_{k}(x)=s, \operatorname{dim}_{k(u, r)}(x)=t$, then $\operatorname{dim}_{k}(u, v, x)=\operatorname{dim}_{k}(u, x)$ $=N+1+t$, hence $\operatorname{dim}_{k:(r)}(u)=N+1+t-s$. Since the point $P=(x)$ lies on $V$, we have $s \leqq r$, and $N+1+t-s \geqq N+1+t-r \geq N-r+1$, i. $e$. there are at least $(N-r+1)$ independent variables over $k(x)$ among $u_{i}(i=1, \ldots \ldots, N)$. Since the rank of the matrix $A$ is $N-r$, without loss of generality we can assume that

$$
\left|\frac{\partial G_{v}}{\partial x_{i}}\right| \geqslant 0 \quad\left(\begin{array}{l}
\nu=1, \ldots \ldots, N-r \\
i=1, \ldots \ldots, N-r
\end{array}\right) .
$$

Suppose that the rank of the matrix $B$ is at most equal to $N-r$, then we have the relations ${ }^{2)}$

$$
\left|\begin{array}{ccc}
\frac{\partial G_{1}}{\partial x_{1}} \ldots \ldots & \ldots \frac{\partial G_{1}}{\partial x_{N-r}} & \frac{\partial G_{1}}{\partial x_{k}} \\
\ldots \ldots \ldots \ldots \ldots \ldots \ldots & \ldots \ldots \ldots \ldots, N) \\
\frac{\partial G_{N-r}}{\partial x_{1}} \ldots & \frac{\partial G_{N-r}}{\partial x_{N-r}} & \frac{\partial G_{N-r}}{\partial x_{k}} \\
u_{1} \ldots \ldots & u_{\dot{N}-r} & u_{k}
\end{array}\right|=0 \quad(k=N-r+1, \ldots, N)
$$

and $u_{k}(k=N-r+1, \ldots, N)$ can be expressed as the linear combinations of $u_{i}(i=1, \ldots, N-r)$ with the coefficients in $k(x)$. It contradicts to the preceding fact.

Thus we get the

THEOREM. Let $P$ be a point on the generic hyperplane section $W^{r-1}$ of an algebraic variety $V^{r}(r>1)$. Then if $P$ is simple on $V, P$ is also simple on $W$. 


\section{Notes}

1) This can be shown easily using the Lemma, due to Mr. T. Matsusaka, which is a generalization of Zariski's Lemma. O. Zariski. "Pencils on an algebraic variety and a new proof of a theorem of Bertini." Trans. A. M. Soc. 1941, p. 68, Lemma 5. T. Matsusaka. "The theorem of Berini on linear systems in modular fields." Mem. of the College of Science, Univ. Kyôto Vol. XXVI, Math. No. 1, 1950, p. 55. th. 2. 4 .

2) For the device of the following proof I thank to my friend Mr. Hajime Nishimura.

Added in the proof. I find in his letter to Mr. J. Igusa of Nov. 3, 1950, that Prof. Weil had already obtained this result as an important lemma in the generaI theory of the Picard variety. (Nov. 25, 1950.) 